\title{
COMT Inhibitors in Parkinson's Disease
}

\author{
Jean Rivest, C. Lynn Barclay and Oksana Suchowersky
}

\begin{abstract}
The COMT inhibitors, tolcapone and entacapone, are a new class of Parkinson's medications. By inhibiting the enzyme catechol-o-methyl-transferase (COMT), they prevent peripheral degradation of levodopa, allowing a higher concentration to cross the blood-brain barrier. Pharmacokinetic studies have shown that both tolcapone and entacapone significantly prolong the elimination half life, and increase the area under the curve of levodopa without increasing $\mathrm{C}$ max. Clinical studies with COMT inhibitors have shown benefit in both stable and fluctuating PD patients with improvement in motor function with lower levodopa doses. Fluctuating patients also had increased "on" time and reduced "wearing off". Side effects were most commonly related to increased dopaminergic stimulation. Specific side effects included diarrhea and elevated liver enzymes. The recent reports of three cases of fulminant hepatitis with the use of tolcapone has led many countries to remove this compound from their market. Concerns about a possible class effect should impose close monitoring of liver function tests with the use of any of the nitrocatechols.
\end{abstract}

\begin{abstract}
RÉSUMÉ: Inhibiteurs de la COMT dans la maladie de Parkinson. Les inhibiteurs de la COMT, le tolcapone et l'entacapone, appartiennent à une nouvelle classe de médicaments antiparkinsoniens. En inhibant l'enzyme catécholo-méthyl-transférase (COMT), ils empêchent la dégradation périphérique de la lévodopa permettant ainsi à des concentrations plus élevées de traverser la barrière hémato-encéphalique. Des études de pharmacocinétique ont montré que le tolcapone et l'entacapone prolongent significativement la demi-vie et augmentent l'aire sous la courbe de la lévodopa sans augmenter le Cmax. Les études cliniques avec des inhibiteurs de la COMT ont montré des bénéfices tant chez les patients stables que chez ceux qui présentent des fluctuations, avec une amélioration de la fonction motrice avec des doses inférieures de lévodopa. Les patients fluctuants ont également présenté une augmentation du temps où ils sont fonctionnels et une diminution de la perte d'effet. Les effets secondaires étaient le plus souvent reliés à l'augmentation de la stimulation dopaminergique. La diarrhée et une élévation des enzymes hépatiques sont des effets secondaires spécifiques de ces médicaments. Trois cas d'hépatite fulminante avec le tolcapone ont incité plusieurs pays à retirer ce produit du marché. À cause de la possibilité qu'il s'agisse d'un effet de classe, on doit faire une surveillance étroite des épreuves de fonction hépatique dans l'utilisation de tous les nitrocatéchols.
\end{abstract}

Can. J. Neurol. Sci. 1999; 26: Suppl. 2-S34-S38

\section{COMT Inhibitors in Parkinson's Disease}

The clinical manifestations of Parkinson's disease (PD) are primarily a reflection of the nigrostriatal dopamine deficiency. More than 30 years after it was demonstrated that high dose of Ldopa could produce substantial clinical improvement, L-dopa remains the mainstay of therapy of this condition.

\section{MetABolisM OF L-DOPA AND THE RATIONALE OF COMT INHIBITION}

L-dopa is absorbed predominantly in the proximal small bowel. ${ }^{1}$ Its uptake depends on an active transport system, in competition with other large neutral amino acids. The rate of gastric emptying is a key factor in the absorption of the drug; anticholinergics, large meals and low gastric $\mathrm{pH}$ will delay delivery of L-dopa to the duodenum, resulting in reduced and delayed peak plasma concentrations. In the fasting state, peak plasma concentrations are observed within 1 or 2 hours, and elimination half-life ranges from 0.78 hours to 1.74 hours. It must be emphasized that the overall pharmacokinetics of L-dopa are not modified by the progression of the disease.

L-dopa is metabolised in the periphery by aromatic acid decarboxylase (AAAD) and catechol-O-methyltransferase (COMT), the former being the most important (Figure 1). In current practice, L-dopa is administered with peripheral dopa-decarboxylase inhibitors (carbidopa, benzerazide) which do not cross the blood-brain barrier. The major mechanism of dopa conservation is inhibition of gut wall decarboxylase. The coadministration of AAAD inhibitors with L-dopa allows a reduction of 70 to $80 \%$ in total daily L-dopa dose required to produce clinical benefit. The peripheral production of dopamine is therefore largely

From the Centre Universitaire de Santé de l'Estrie, Fleurimont, Quebec (JR), University of Ottawa, Ottawa, Ontario (CLB), University of Calgary, Calgary, Alberta (OS) Reprint Requests to: Dr. Jean Rivest, Centre Universitaire de Santé de l'Estrie, 3001, 12th Avenue North, Fleurimont, Quebec, Canada JlH 5N4 


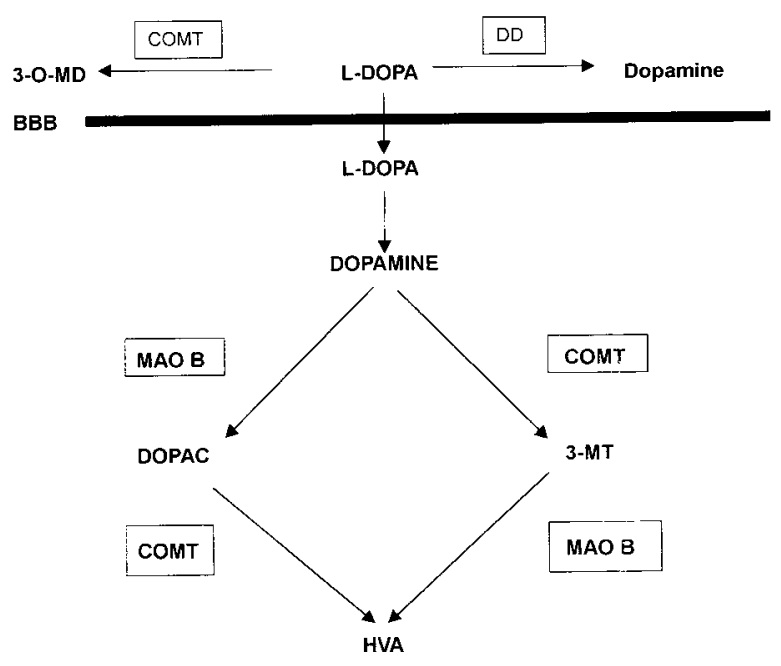

DOPA-Dihidroxyphenylalanine

3-O-MD-3-0-Methyldopa

BBB-blood brain bamer

DOAPC-Dihydroxy Pthenylacetic acid

HVA-homovanillic acio

3MT-3-Methoxythyramine

Figure 1: Synthesis of dopamine and its metabolism by cathechol-omethyl transferase (COMT) and monoamine oxidase (MAO).

reduced, along with the side effects such as nausea, vomiting and arrythmias. However, the plasma half-life of L-dopa remains unaffected.

With the blockade of AAAD, COMT becomes the main metabolic pathway, transforming L-dopa into an inactive metabolite, 3-O-methyldopa (3-OMD). ${ }^{2}$ With L-dopa combination therapy, $3-O M D$ is the major plasma metabolite with plasma concentrations largely exceeding those of L-dopa. COMT is widely distributed, notably in the gut wall, liver, kidneys, muscles and even in the endothelium of the blood-brain barrier. It is also present in the brain, where O-methylation of dopamine is one means of terminating its action. The blockade of this enzyme could be advantageous in many ways. Increased absorption of L-dopa and reduction of hepatic metabolism would increase bioavailability of L-dopa. Reduction of O-methylation of L-dopa in the systemic circulation would prolong its half-life and increase the area under the curve. Central inhibition of COMT would inhibit degradation of dopamine (Figure 1).

The reduction in the peripheral formation of 3-OMD and of the brain dopamine degradation, is probably not of clinical significance. High levels of 3-OMD, a neutral amino acid could in theory have a negative impact on the clinical response to L-dopa by competing with its transport through the intestinal mucosa and the blood-brain barrier. However, 3-OMD represents only about $10 \%$ of the neutral amino acids which all might compete with L-dopa. The significant reduction of plasma 3-OMD concentrations after 8 weeks of treatment with entacapone, a peripheral COMT inhibitor, did not prolong the response duration to Ldopa, arguing against any significant impact of elevated plasma 3-OMD on the pharmacokinetics of L-dopa. ${ }^{3}$

It is unclear whether intracerebral COMT blockade is of clinical significance since the administration of tolcapone (a COMT inhibitor acting also centrally) without L-dopa to parkinsonian patients did not provide any detectable clinical benefits (how- ever, the dose of tolcapone in this trial may have been too low to cross the blood-brain barrier). ${ }^{4}$

\section{COMT INHIBITORS: THE NITROCATECHOLS}

Previous clinical experience with COMT inhibitors has been disappointing, owing mostly to the absence of specific and potent oral compounds. The situation has changed with the recent development of the nitrocatechols, a group of highly potent COMT inhibitors including nitecapone, entacapone and tolcapone. These compounds are highly selective, with virtually no action on tyrosine hydroxylase, dopamine B-hydroxylase, dopa decarboxylase, and monoamine oxidase A and B.

In rats with unilateral nigral lesions, oral nitecapone combined with L-dopa/carbidopa increases the striatal levels of levodopa, dopamine, DOPAC and HVA in a dose-dependent fashion, correlating with the observed behavioural changes. ${ }^{2}$ At the doses studied, nitecapone appears to mainly inhibit the duodenal COMT without affecting the striatal COMT. In preliminary experiments, no entry of radioactive nitecapone into the brain was found. There is little data on human subjects with PD, with only one preliminary study describing "significant reduction of disability" in all 7 patients studied. ${ }^{5}$

Entacapone is a reversible inhibitor of COMT acting primarily in the periphery. The plasma elimination half-life of the drug is 1.5 to 3.5 hours. In normal volunteers, single oral doses of 100 to 400 $\mathrm{mg}$ inhibit red blood cell COMT by 50 to $75 \%$. In human parkinsonian subjects, the coadministration of $1200 \mathrm{mg}$ daily of entacapone with L-dopa/carbidopa preparations prolongs the elimination half-life of L-dopa by $59 \%$ to $75 \%$, and increases the area under the curve AUC by $45 \% .^{3}$ There is, however, no significant change of Cmax and Tmax, indicating that entacapone has little effect on absorption and first-pass metabolism of L-dopa despite the high concentrations of COMT in the gut and liver. Concentrations of 3-OMD on chronic entacapone therapy are reduced by almost $60 \%$, but rise rapidly on the day the drug is discontinued, in agreement with the reversible nature of this inhibition.

Tolcapone is the most potent and selective COMT inhibitor identified thus far. The drug is rapidly absorbed (Tmax $1.5 \mathrm{hrs}$ ) and shows dose-proportional pharmacokinetics. It has a mean plasma elimination half-life of $2.0+/-0.8$ hours, and must be administered in 3 divided doses. ${ }^{6}$ The metabolism is primarily hepatic. The onset of COMT inhibition is less than 2 hours in most subjects. The degree of inhibition increases with increasing dose, attaining $80 \%$ with a single dose of $100 \mathrm{mg}$. Like entacapone, tolcapone appears to have little impact on L-dopa firstpass metabolism since, up to a dose of $200 \mathrm{mg}$, the $\mathrm{C}$ max and $\mathrm{t}$ max of L-dopa are unchanged. Coadministration of Ldopa/benserazide with increasing doses of tolcapone prolongs Ldopa elimination half life and increases the AUC by a similar ratio, of about twofold. This occurs with doses of $100 \mathrm{mg}$ and $200 \mathrm{mg}$ t.i.d. ${ }^{7}$ Further increases of the tolcapone dose provides no additional benefit on L-dopa pharmacokinetics. In fact, higher doses prolong L-dopa t max, possibly by competing for the saturable transport mechanism across gut membranes.

\section{COMT INHIBITORS: CLINICAL STUDIES}

The addition of COMT inhibitors to L-dopa preparations significantly prolongs levodopa elimination half life without 
increasing peak plasma concentrations. This enhanced, less pulsatile L-dopa delivery to the brain has been clearly demonstrated in various pharmacokinetics studies. During chronic entacapone treatment in patients with PD, the mean plasma L-dopa concentrations were increased by $23 \%$ (despite a $27 \%$ reduction in daily L-dopa dosages) and the variation of plasma L-dopa levels throughout were reduced from $49 \%$ to $38 \%$. $^{3}$

The potential beneficial effects of this group of compounds in PD are numerous. By permitting a more sustained, physiological dopaminergic stimulation, they could help prevent the development of motor complications, since both animal and human experiments suggest that intermittent dopaminergic stimulation is a key factor for the induction of dyskinesia and shortening of response. ${ }^{8}$ A 5 year clinical study using tolcapone in patients with a stable motor response on levodopa therapy was initiated to address this issue, but had to be interrupted due to recent reports of fulminant hepatitis in three patients (see further in text).

Patients already experiencing motor fluctuations should, however, get the most immediate benefit. The prolonged plasma elimination half-life is expected to prolong the therapeutic effect of each L-dopa dose, therefore increasing "on" time. The increase in mean through L-dopa plasma levels could also attenuate the severity of "off" periods. A reduction in the number of daily L-dopa intakes and of the total dosage is also anticipated.

\section{A) Stable patients}

The North American trial on the efficacy and safety of tolcapone in stable patients was conducted in 20 centres and included a total of 298 patients. ${ }^{9}$ Individuals aged at least 30 years with a secure diagnosis of PD and without evidence of motor fluctuations or significant dyskinesias were eligible. All patients were receiving L-dopa/carbidopa preparations for at least 3 months (maximum of 5 years), with dosage ranging from $100 \mathrm{mg}$ to 600 $\mathrm{mg}$ daily. Exclusion criteria included the following: use of dopamine agonists during the previous 4 weeks, long-term treatment with centrally acting dopamine antagonists during the preceding 6 months, MAO inhibitors (other than selegiline), depression and clinically relevant active medical problems.

Patients were randomly assigned to receive placebo, tolcapone $100 \mathrm{mg}$ t.i.d., or tolcapone $200 \mathrm{mg}$ t.i.d. Assessments included United Parkinson's Disease Rating Scale (UPDRS) scores, L-dopa induced symptoms, change in total daily L-dopa dosage, and Sickness Impact Profile (SIP), a generic measure of quality of life. Both treatment groups showed a statistically significant reduction of UPDRS subscale II, indicating improved functioning for activities of daily living. The effect of tolcapone was greatest in those with the most marked impairment at baseline $($ score $>6$ ). There was also a significant reduction of UPDRS motor score (subscale III) in both treatment groups, with the greatest improvements in those with the highest scores at baseline. These improvements were maintained up to the 12 months assessment. The SIP scale showed a significant improvement only in physical scores. A slight but statistically significant reduction of daily L-dopa dosage was observed in both treatment groups at 6 months, compared to the placebo group. Overall, the improvements were marginally greater in the $200 \mathrm{mg}$ t.i.d. treatment group. Fourteen patients in the placebo group required additional antiparkinsonian medication (most often dopamine agonists) at 6 months, compared to only 2 in each tolcaponetreated group. The development of motor fluctuations was more common in the placebo group: $26 \%$, compared with $14 \%$ in the tolcapone $200 \mathrm{mg}$ t.i.d. group, and $19 \%$ in the $100 \mathrm{mg}$ t.i.d. group (not significant).

Most adverse events were mild in severity. Dopaminergic effects (nausea, anorexia, dyskinesia, sleep disorders) were predictably more common in the tolcapone-treated patients, especially the $200 \mathrm{mg}$. t.i.d group. These tended to develop early in the course of treatment but were rarely a reason for withdrawal from the study. Diarrhea was the most frequent nondopaminergic adverse event, leading to eight withdrawals in the tolcapone 100 $\mathrm{mg}$ t.i.d. group and five in the $200 \mathrm{mg}$ t.i.d. group (compared to one in the placebo group). In most cases, the diarrhea began 30 to 90 days after starting treatment, and resolved when the drug was discontinued. Raised liver enzymes ( $>3$ times the upper normal limit) was the only laboratory abnormality seen, occurring in $3 \%$ of the $100 \mathrm{mg}$ t.i.d. group, and $5 \%$ of the $200 \mathrm{mg}$ t.i.d. group. Four patients were withdrawn while in four others the values returned to normal while on treatment. All cases developed within 1 to 6 months of starting treatment.

\section{Comments:}

The improvements in UPDRS subscales II and III were statistically significant, yet clinically modest. Patients most severely impaired benefited the most, implying that the benefit was marginal for many patients with mild disease. Of interest is the observation that, at 6 months, fewer of the tolcapone-treated patients required additional medication and had developed motor fluctuations. One could postulate that a proportion of "stable" patients were in fact mild fluctuators, and these are likely to benefit from a prolongation of the elimination half-life of L-dopa. The authors from the study admitted that $18 \%$ to $25 \%$ of the patients had mild wearing-off, defined as "off"-periods accounting for less than $25 \%$ of the waking day.

At the present time, the value of COMT inhibitors in patients with mild, stable disease is arguable, considering the modest clinical benefits, the added costs and the potential for additional adverse effects.

\section{B) Patients with motor fluctuations}

The effects of entacapone on the pharmacokinetics of L-dopa and the clinical response to L-dopa was assessed in an open-label trial in 15 patients with PD and motor fluctuations. ${ }^{3}$ On chronic entacapone treatment with $1200 \mathrm{mg}$ daily (400 mg t.i.d.), the daily L-dopa doses were reduced by $27 \%$ in response to patient's report of increased dyskinesia or of lesser requirements for L-dopa. Despite this reduction, the mean L-dopa concentration were $23 \%$ higher than that during the run-in phase. The L-dopa concentration in the morning before the first dose of Sinemet of the day was, however, unchanged, indicating that there was no accumulation of L-dopa from one day to the next. The time spent "on" increased from $63 \%$ to $77 \%$. Many patients reported reduce "off" dystonia and increased sense of well-being. Increased dyskinesia was common but easily managed by reducing L-dopa doses. Diarrhea occured in 3 patients, and caused one drop out of the study.

In two multicenter, placebo-controlled trials, ${ }^{11,12}$ patients were randomized to receive either entacapone $200 \mathrm{mg}$ or matching placebo with each dose of levodopa and were followed for 24 weeks. In both studies, time spent "on" increased of approximately one hour/day (about 10\%) and this effect was consistent across time. Daily levodopa dosage was reduced by about $13 \%$. 
Mild dopaminergic side effects, including dyskinesias subsided after adjustment of levodopa dosage. No change has been detected in liver function tests or other laboratory safely parameters.

The clinical experience with the chronic use of tolcapone in patients with PD presenting motor fluctuations is more impressive, with the recent publications of three multicenter trials totalizing 530 patients. Kurth et al. performed two 10-hour clinical evaluations at baseline and day 42 comparing placebo and one of three tolcapone doses (50mg, $200 \mathrm{mg}, 400 \mathrm{mg}$ ) on a total of 151 patients. ${ }^{13}$ Tolcapone significantly reduced "off" time an average of $40 \%$ and increased total "on" time by about $25 \%$ at all doses levels, compared to the placebo group. L-dopa daily dosage was significantly reduced, especially in the $200 \mathrm{mg}(26 \%)$ and 400 mg $(24 \%)$ groups.

Rajput et al. performed a randomized, double-blind, parallelgroup trial at 11 centers in North America, comparing tolcapone $100 \mathrm{mg}$ and $200 \mathrm{mg}$ t.i.d. with placebo in a total of 202 patients. ${ }^{14}$ The primary aim was to measure the effect on the "wearing-off" phenomenon, using patients' self-rating diaries. Other efficacy measures included the investigators' global assessment (IGA) and UPDRS subscales I to IV. With both dosages of tolcapone, the duration of daily "off" time was reduced from baseline, but the change was significant only in the $200 \mathrm{mg}$ t.i.d. group. At the end of month 3 , the patients in this group of treatment had a mean 3.25 hours reduction in daily "off" time. The dosage of L-dopa was significantly reduced in both treatment groups, by about $22 \%$. The IGA of the "wearing-off" phenomenon indicated that $68 \%$ of patients receiving tolcapone $100 \mathrm{mg}$ t.i.d. and $95 \%$ of those on $200 \mathrm{mg}$ t.i.d. had a reduced "wearing-off" compared with $37 \%$ of those on placebo. No significant differences were seen between the groups in UPDRS total and subtotal scores for subscales I,II and III. With this particular trial design, about $30 \%$ of patients completed 12 months of treatment. The reductions in L-dopa dosage and "off" time seen at the end of month 3 were maintained in the tolcapone-treated patients.

A similar multicentre trial was also conducted in Europe, involving a total of 177 patients. ${ }^{15}$ At the end of 3 months, treatment with tolcapone significantly decreased mean "off" time (by $>20 \%$ ) and increased mean "on" time (by $>25 \%$ ) compared to placebo. Both tolcapone dosages were equally effective in increasing "on" time, but the $100 \mathrm{mg}$ t.i.d. dosage was more effective in decreasing "off" time. The decrease in "off" time was maintained at 9 months in both treatment groups. The mean reduction of L-dopa dosage at month 3 , in the order of $23 \%$, was significant in both groups but maintained at 9 months in the 200 mg t.i.d. group only.

The tolerability in all 3 studies was comparable. Dopaminergic adverse effects including dyskinesia, were more common in both treatment groups, notably in the $200 \mathrm{mg}$ t.i.d. group, and occurred early in the study. They were usually transient and could be satisfactorily controlled by L-dopa dosage adjustment. Dyskinesia were never the reason for withdrawal of the studies. Diarrhea was the most often reported non-dopaminergic adverse event and the most often cited reason for withdrawal, causing $5 \%$ to $6 \%$ of patients to drop out of the studies. The mechanism is unknown, and the incidence unrelated to tolcapone dosage. Most cases occur between day 30 and 90 after starting treatment, and it is always reversible after discontinuation of tolcapone (rechallenge with tolcapone in these individu- als always results in diarrhea recurrence (OS, unpublished observation). Asymptomatic elevation of liver transaminases is observed in $2 \%$ to $3 \%$ of patients. The enzyme concentrations return to normal values when treatment is withdrawn, although in some patients the values have returned to normal while on continued treatment.

More recently, three patients have presented a fulminant hepatitis while on tolcapone, leading many countries to withdraw this compound from their market. In all three cases, the hepatitis occurred during the first six months of treatment, and no other cause was found on investigation. Histological findings from liver biopsy in case one were consistent with drug-related hepatotoxicity. ${ }^{16}$ The causal relationship remains under investigation at the present time.

Another rare complication associated with the use of tolcapone is the neuroleptic malignant syndrome (NMS). A too agressive reduction of dopaminergic agents while introducing tolcapone, or its abrupt discontinuation may precipitate this potentially lethal complication. A reduction of about 25 to $30 \%$ in daily levodopa dose is recommended when introducing tolcapone. Conversely, levodopa or dopamine agonists doses must be increased when discontinuing tolcapone. Rhabdomyolysis, either in the setting of NMS or severe dyskinesia, has also been reported.

\section{Comments}

The efficacy of COMT inhibitors for the control of motor fluctuations reflects the significant impact of this group of compounds on L-dopa pharmacokinetics. The effects are often noticed within 24 hours of drug introduction, owing to the rapid onset of COMT inhibition. In order to prevent dopaminergic side effects, it is recommended to reduce the daily dosage of L-dopa by about $25 \%$ when tolcapone is introduced in patients who are experiencing significant dyskinesia. Both dosages of tolcapone (100 mg t.i.d. and $200 \mathrm{mg}$ t.i.d.) are effective in reducing "off" time and improving "on" time, but have no significant impact on the magnitude of the motor response during the "on" period. Recent reports have suggested that there is no significant difference between the $100 \mathrm{mg}$ or $200 \mathrm{mg}$ doses in most patients. ${ }^{17,18}$ Therefore, the recommended starting dose of tolcapone is 100 $\mathrm{mg}$ t.i.d., increasing to $200 \mathrm{mg}$ t.i.d. only if a satisfactory response is not attained. Warning patients of the possibility of worsening of dyskinesia is important as is maintaining patient physician contact in the first few days of initiating treatment.

At the present time, tolcapone is no longer commonly available in many countries or must be used under strict regulations, with close monitoring of liver function tests. No laboratory abnormalities have yet been reported with entacapone, but the number of patients studied is considerably smaller. Concerns about a possible class effect should command serial measurements of serum biochemistry, at least for the first six months of treatment, with any of the nitrocatechols.

\section{Conclusion}

COMT inhibitors extend levodopa's duration of action, prolonging "on" time in patients with motor fluctuations. They provide rapid-onset improvement and may be preferred in the older age group over dopamine agonists, more likely to induce adverse CNS effects (psychosis, hallucinations) and orthostatic hypotension. ${ }^{19}$ On the other hand, younger patients and those with 
prominent dyskinesias may be better served by dopamine agonists. The combination of COMT inhibitors with dopamine agonists will likely also provide additional benefit in severe fluctuators but requires further investigation.

\section{REFERENCES}

1. Cedarbaum J. Clinical pharmacokinetics of anti-parkinsonian drugs. Clin Pharmacokinet 1987; 13: 141-178.

2. Mannistö PT, Kaakkola S. Rationale for selective COMT inhibition as adjuncts in the drug treatment of Parkinson's disease. Pharmacol Toxicol 1990; 66: 317-323.

3. Nutt JG, Woodward WR, Becker RM, et al. Effects of peripheral catechol-O-methyltransferase inhibition on the pharmacokinetics and pharmacodynamics of levodopa in parkinsonian patients. Neurology 1994; 44:913-919.

4. Hauser RA, Molho E, Shale H et al. A pilot evaluation of the tolerability, safety and efficacy of tolcapone alone and in combination with oral selegeline in untreated Parkinson's disease patients. Mov Disord 1998; 13: 643-747.

5. Teräväinen H, Kaakkola S, Järvinen M, Gordin A. Selective COMT Inhibitor, Nitecapone, In: eds Parkinson's disease. 42nd Annual Meeting of the American Academy of Neurology, Miami Beach, Florida, 1990 (abstract)

6. Dingemanse J, Jorga KM, Schmitt M, et al. Integrated pharmacokinetics and pharmacodynamics of the novel catechol-O-methyltransferase inhibitor tolcapone during first administration to humans. Clin Pharmacol Ther 1995; 57: 508-517.

7. Dingemanse J, Jorga KM, Zürcher G, Schmitt M, Sedek G, Prada $\mathrm{MD}$ et al. Pharmacokinetic-pharmacodynamic interaction between the COMT inhibitor tolcapone and single-dose levodopa. Br. J. Clin Pharmacol 1995; 40: 253-262.

8. Nutt JG. Levodopa: rational and irrational pharmacology. Ann Neurol 1994; 36: 4-5.

9. Waters $\mathrm{CH}$, Kurth $\mathrm{M}$, Bailey $\mathrm{P}$, et al. Tolcapone in stable Parkinson's disease: efficacy and safety of long-term treatment. Neurology 1997; 49: 665-671.
10. Suchowersky O, Martin WRW, Bailey et al. Benefit of tolcapone in patients with early Parkinson's disease. Neurology 1996; 46 (Suppl 2): A 159.

11. Rinne UK, Larsen JP, Siden A, Worm-Petersen J and the NomeCOMT Study Group. Entacapone enhances the response to levodopa in Parkinsonian patients with motor fluctuations. Neurology 1998; 51: 1309-1314.

12. Parkinson Study Group. Entacapone improves motor fluctuations in levodopa treated Parkinson's disease patients. Ann Neurol 1997, 42: 747-755.

13. Kurth $\mathrm{MC}$, Adler $\mathrm{CH}$, St-Hilaire $\mathrm{M}$, et al. Tolcapone improves motor function and reduces levodopa requirements in patients with Parkinson's disease experiencing motor fluctuations: a multicentres, double-blind, randomized, placebo-controlled trial. Neurology 1997; 48: 81-87.

14. Rajput AH, Martin W, Saint-Hilaire M-H, Dorflinger E, Pedder S. Tolcapone improves motor function in the Parkinsonian patients with the "wearing-off" phenomenon: a double-blind, placebocontrolled, multicenter trial. Neurology 1997; 49: 1066-1071.

15. Baas H, Beiske AG, Ghika J, Jackson M, Oertel WH, Poewe W \& al. Catechol-O-methyltransferase inhibition with tolcapone reduces the "wearing-off" phenomenon and levodopa requirements in fluctuating parkinsonian patients. J Neurol Neurosurg Psychiatry 1997; 63: 421-428.

16. Guttman $M$ and The Canadian Tolcapone Fluctuating Study Group. Comparison of tolcapone $100 \mathrm{mg}$ vs $200 \mathrm{mg}$ in fluctuating Parkinson's Disease (PD) patients. Mov Disord 1998; 13 (Suppl 2): 175.

17. Suchowersky O, Panisset M, Bailey P et al. Comparison of two different doses of tolcapone in non-fluctuating Parkinson's disease patients. Mov Disord 1998; 13 (Suppl 2): 175

18. Assal F., Spahr L, Hadengue et al. Tolcapone and fulminant hepatitis. Lancet 1998; 352: 9132.

19. Agid A, Destée A., During F, Monstatruc JL, Pllack P on behalf of the French Tolcapone Study Group. Tolcapone, bromocriptine and Parkinson's disease. Lancet 1997; 350: 712-713. 